\title{
Improve Design and Analysis of Friend-to-Friend Content Dissemination System
}

\author{
Dr. V. Senthil kumar', Mr. P. Jeevanantham², Dr. A. Viswanathan ${ }^{3}$, \\ Dr. Vignesh Janarthanan ${ }^{4}$, Dr. M. Umamaheswari ${ }^{5}$, Dr. S. Sivaprakash ${ }^{6}$ \\ 'Associate Proferssor, Department of CSE, \\ Malla Reddy Institute of Technology and Science, Secunderabad, \\ Email: senthilkmr777@gmail.com \\ 2P.G Scholar, \\ ${ }^{3}$ Proferssor, Department of CSE, \\ Malla Reddy Institute of Technology and Science, Secunderabad, \\ 4 Proferssor, Department of CSE, \\ Malla Reddy Institute of Technology and Science, Secunderabad \\ ${ }^{5}$ Associate Proferssor, Department of CSE, \\ Malla Reddy Institute of Technology and Science, Secunderabad \\ ${ }^{6}$ Proferssor, Department of IT, CMR Engineering College, Secunderabad
}

\begin{abstract}
In this paper, examination another creamer substance storing and scattering structure for customer made substance (UGC). Despite the obviousness of developing the combination plot that manhandles the potential gains of sharp associations, offloading and decentralized limit and transport, to date such an arrangement has not yet been proposed again due to two fundamental reasons. Regardless, taking into account the extraordinary correspondence and energy cost of decentralized substance storing and transport for flexible systems. Second, due to the area these two head limitations by abusing the opportunity of substance replication, considering starting experience time and range of customers' encounters, and utilizing relational collaboration organizations for guileful dispersing. The current structure game plan makes a dispersed decentralized limit system with canny substance replication, which decreases convenient data traffic and gives the customers full control at immaterial cost which can be used to give flexible individual to individual correspondence organizations. Regardless, the results show that it is unbelievable to dependably recognize most powerful customers inside a neighborhood advance transport as they are astoundingly environment subordinate. In this paper extension to online casual associations (OSNs) has experienced tremendous improvement lately. These OSNs offer appealing techniques for electronic social affiliations and information sharing, yet likewise raise different security and insurance issues. Moreover, it A3P separates what the procedure can mean for the suitability of a plan based structure that supports redesigned web access functionalities, like substance filtering and revelation, taking into account tendencies demonstrated by end customers.
\end{abstract}

Keywords: Security Model, Content Dissemination, Model, Adaptive Policy Prediction (A3P), Online casual networks.

\section{Introduction}

In OSNs, information filtering can moreover be used for a substitute, more sensitive, reason. This is a direct result of the path that in OSNs there is the opportunity of posting or commenting various posts on explicit public/private zones, acquired regular dividers. Information filtering can hence be used to empower customers to normally control the messages made on their own dividers, by filtering through unwanted messages. It is acknowledged that this is a key OSN organization that has not been surrendered as of recently. The proposed structure familiarizes three new upgrades with security anagement models:

Assisted Friend Grouping a steady improvement to standard social occasion based game plan the chiefs.

$>$ Same-As Policy Management another perspective improvement over ordinary get-together based methodology the board.

Example Friend Selection a consistent improvement to Same-As Policy Management.

The A3P system handles customer moved pictures, and factors in the going with measures that influence one's security settings of pictures: The impact of social environment and individual characteristics, Social setting of customers, for instance, their profile information and relationship with others may give accommodating information concerning customers' insurance tendencies. For example, customers propelled by photography may get a kick out of the 
opportunity to impart their photos to other fledgling photographic craftsmen. Customers who have a couple of family members among their social contacts may grant to them pictures related to family events.

Nevertheless, using essential plans across all customers or across customers with similar credits may be too foolhardy and not satisfy solitary tendencies. Customers may have drastically different speculations even on comparative kind of pictures. For example, an insurance threatening individual may share all his own photos while a more moderate individual may just have to share singular pictures.

The piece of picture's substance and metadata, all things considered, equivalent pictures routinely cause near security tendencies, especially when people appear in the photos. The A3P-focus highlight on taking apart every individual customer's own photos and metadata, while the A3P-Social offers a neighborhood of perspective on security setting recommendations for a customer's potential insurance improvement. To design the association flows between the two construction squares to change the benefits from meeting singular credits and getting neighborhood. The major objective of this paper is,

$>$ To present a customer helped buddy gathering instrument that overhauls regular social event based procedure the chiefs moves close.

$>$ To found quantifiable comprehension among packs and customer described relationship social events. Additionally, customer perspective on the redesigns should engage.

$>$ To present another security the heads model that is an improvement over standard social affair based course of action the board moves close.

$>$ To impact a customer's memory and appraisal of their allies to set systems for other near associates, which we suggest as Same-As Policy Management.

$>$ To recognize customer security appraisal that can be used to extra improve insurance the heads models.

\section{Related Works}

Abdolreza Abhari and Mojgan Soraya [I] propose a novel common system subject to casual association, where friends are redistributing the accounts that they have saved. A prefetching philosophy is used to prefetch the beginning piece of the accompanying video in the associated casual association in each ally to lessen the start up delay of P2P overlay. Casual associations existing among YouTube accounts are made by social events of related chronicles, in case one video has been watched, another video inside the get-together will undoubtedly be gotten to.

Marco Valerio Barbera and Julinda Stefa [2] presents Extensive examinations with a couple of certifiable and produced enlightening lists show the suitability of our methodologies in offloading: VIP sets of about $7 \%$ and $1 \%$ of association centers in independently grounds like and vehicular adaptability circumstances are adequate to guarantee about $90 \%$ of association offload. Additionally, the presentation of the VIPs picked by our procedures is close to an ideal benchmark VIPs set handled from the full data on the system (i.e., considering past, present, and future contacts among centers). Finally, in this work we revolve around $90 \%$ association incorporation since we tentatively saw that this value prompts a fair congruity among consideration and number of VIPs. Regardless, further assessment of the trade off among consideration and VIP number will be investigated in future work.

Raul Gracia-Tinedo et al.,[3] In this paper they considered Friend-to-Friend (F2F) accumulating systems are nowadays an interesting investigation subject and they set up an elective method to manage impact singular storing. The F2F perspective relies upon the cooperation between casual associations and limit structures: customers store their data in a lot of social colleagues. Consequently, data is neither taken care of in a united specialist nor in dark companions, enabling customers to hold the control of their data. Likewise, the social section of F2F systems mitigates various annoying issues present in huge degree scattered structures for instance security, trust, inspirations.

Dish Hui et al., [4] In this paper is base on two unequivocal pieces of society: neighborhood centrality. Neighborhood a critical characteristic of PSNs. Investment ties, yet moreover parcels human culture into networks. Human culture is coordinated. Inside a neighborhood, not many gathering are more standard, and speak with a more noteworthy number of people than others (i.e.have high centrality); we call them focus focuses. Notoriety situating is one piece of the general population. For an ecological neighborhood, collaboration suggests that a day to day existence type of a given kind is bound to interface with another animal of a comparable sort than with a subjectively picked individual from the general population. This related coordinated effort thought moreover applies to human, so we can mishandle such a neighborhood to pick sending ways.

Azeem J. Khan et al., [5] propose another notification transport structure called CAMEO, to pleasantly lighten the pressing factor from such unnecessary traffic. Appearance hopes to expect customer setting and as such proactively recognize appropriate publicizing substance, and a while later shrewdly use un-metered or modest far off associations to 
perceptively save promotion content on the PDA. Appearance can achieve extensive impact and enable new sorts of versatile publicizing organizations by using on headways, (for instance, coordinated pre-bringing, pariah obvious status and unequivocal association partition of business traffic).

Anna-Kaisa Pietiläinen and Christophe Diot, [6] In this paper propose a framework to break the common contact graph into lots of centers that meet even more periodically and for longer time spans during the investigation. Maker calls these gatherings brief organizations. The passing neighborhood system proceeds in two phases. In any case, fragment each portrayal outline into more unobtrusive and denser gatherings of center points. Second, we apply a reformist gathering estimation to add up to the portrayal packs into huge organizations.

Kanchana Thilakarathna et al., [7] Decentralized long reach relational correspondence (D-OSN) have been proposed as the best approach to improve customer security and the customer neglecting to keep a hold on their data. Nevertheless, none of these organizations have seen expansive use for different reasons Firstly, because of most customers have adequately purchased in to the C-OSNs. Besides, in light of the fact that D-OSN organizations are unnecessarily jumbled and expensive for a typical customer. Thirdly, in light of the fact that customers are not having the choice to move from their current organizations, due to singular data being gotten with purchased in C-OSNs. Yalut achieves the abovementioned while guaranteeing customer security by getting customer data a long way from untouchable expert communities, using appropriated content transport and limit, conceding up loads, and judicious downloading of substance.

V.Senthilkumar et al,, [8] SDN security audit, will unequivocally investigate the potential It warms of man-in-the-middle attacks on the Open Flow control channel, it also portray an attainable attack model in the open stream channel, and the execution of attack showings to show the limit aftereffects of such attacks.

\section{Procedure}

There are two critical parts in this paper, for instance, Image request and Adaptive technique conjecture. For each customer, his/her photos are first masterminded reliant on substance and metadata [10]. By then, security approaches of each characterization of pictures/substance are penniless down for the plan assumption. For Image request, number of classes are fixed and consigned during picture development, and thereafter portrayal is arranged in those characterizations. By then during looking, these data go about as meta-data for organizing pictures. Given an image, a customer by and large initially finishes up who can get to the image/content, by then examines what express access rights (e.g., see just or download) should be given, ultimately refine the passage conditions, for instance, setting the end date. Correspondingly, the reformist mining first look [II]. Similarly the proposed structure manages parental control based security ensuring in various settings level as well. For example, web content taken may be from more than one picture. So insurance shielding local area arranged naming at whatever point applied to content with various picture, by then it ends up being more feasible to beneficial to end customers [12]. It develops a web application where every one of the recently referenced measures are done along these lines end customers use it.

\section{Group Creation}

The head logins to the site using this module. One of the username and passwords is to be given to login to the application. The chief elective page will be shown exclusively after genuine login. The head adds bundle nuances using this module. The customer enrollment incorporates picking any of the group [13, 14].

\section{Relationship Type Addition}

The head adds relationship type nuances using this module. The customer during other customer development may pick any of the relationship type like Family, Friend, Colleague, etc .[14]

\section{Filtering Rules}

In this stage, an isolating guideline is given. An isolating standard FR is a tuple (maker, creatorSpec, contentSpec, action), where

Author is the customer who shows the norm;

$>$ Creatorspec is a producer assurance.

$>$ Contentspec is a Boolean explanation described on content objectives of the construction $(\mathrm{C}, \mathrm{ml})$, where $\mathrm{C}$ is a class of the first or second level and $\mathrm{ml}$ is the base enlistment level breaking point required for class $\mathrm{C}$ to make the restriction satisfied;

$>$ Action (block, educate) connotes the movement to be performed by the structure on the messages organizing with contentSpec and made by customers recognized by creatorSpec. 
All things considered, in excess of a filtering rule can apply to a comparable customer. A message is in this way disseminated simply in case it isn't deterred by any of the isolating concludes that apply to the message producer. Note also, that it may happen that a customer profile doesn't contain a motivator for the attribute(s) suggested by a FR .[15]

\section{Privacy Settings}

The customer sets assurance nuances using this module. These settings will be the default, yet the customer can change/change the setting during moving the photo as well.

\section{Assisted Friend Grouping}

This fragment is a continuous improvement to standard social affair based course of action the chiefs. It loosens up in two regions: I) outfits the customer with assistance with get-together their sidekicks, and 2) enables the customer to set friend level unique cases inside the get-together system.

For example, when the customer is picked, the customer's degree of relationship with the extra sidekicks are resolved and appeared for each relationship type the customer have[16] .

\section{Same-as Policy Management}

This part is another perspective improvement over standard social affair based system the chiefs. In bundle based methodology the board, the customer ought to at first pack their colleagues.

After which, they ought to pick pack assents (setting the social occasion technique). Finally, partner level extraordinary cases for the social event system are set. A customer's thought (mental model) is occupied with different regions. Where as in Same-As Policy Management, the customer's thought is revolved around a specific buddy [17].

Customers impact their memory and evaluation of an ally to set methodologies for other like associates. Essentially, it uses a buddy affirmation approach, with inconsequential endeavor impedances, to help the customer in setting game plans. Customers are gathered by how they are related with various customers and are appeared in the solicitation [18]. For example, if a given customer's mean relationship (Family) with unexpected customers in comparison to other association transport types, by then the customer has a spot with Family bundle. So all of the customers are resolved reliant on this calculation and keep in those gatherings and are shown so the customer may be place in that particular association transport for the current logged user[19].

\section{Example Friend Selection}

This part is a steady improvement to Same-As Policy Management. Here, it present the customer's associates in bunch demand, i.e., all of the colleagues in Cluster \#I are acquainted with the customer followed by all of the buddies in Cluster\#2, and so on The fundamental partner presented for each gathering is the buddy with the most broad level (buddy with the most significant number of friend relationship) in that bundle [20]. This friend is comparable to show ally for that bundle. The explanation is the particularly related friends are possibly more striking and in this manner more straightforward to review making them incredible competitor for same-as model buddies [2I].

\section{Exploratory Analysis}

\section{A. Worst Case Analysis}

Table 4.I shows the eventual outcome of A3P and Secure Model. The table contains mark simiarity, secure name simiarity and most skeptical situation screw up rate per customer correspondence nuances showed up.

Table 4.I

Worst Case Analysis A3P AND SM

\begin{tabular}{|c|c|c|c|c|}
\hline S.NO & TP & $\begin{array}{l}\text { A3P } \\
\text { USERS } \\
\text { (WC) }\end{array}$ & TP & $\begin{array}{l}\text { Secure Mode (SM) } \\
\text { (WC) }\end{array}$ \\
\hline 1 & 40 & 25.00 & 50 & 20.00 \\
\hline 2 & 50 & 20.00 & 60 & 16.60 \\
\hline 3 & 60 & 16.60 & 70 & 14.28 \\
\hline 4 & 70 & 14.28 & 80 & 12.50 \\
\hline 5 & 80 & 12.50 & 90 & 11.11 \\
\hline
\end{tabular}


Dr. V. Senthil kumar, Mr. P. Jeevanantham, Dr. A. Viswanathan,

Dr. Vignesh Janarthanan, Dr. M. Umamaheswari , Dr. S. Sivaprakash

$$
\mathrm{O}(\mathrm{n})=\mathrm{WA}=\frac{\text { NUMBER OF USERS }}{\text { TOTAL PRILWAGE [TP] }} * 100
$$

Fig 4.I shows the result of A3P and Secure Model [SM]. The table contains tag simiarity, secure tag simiarity and worst case error rate per user communication details shown.

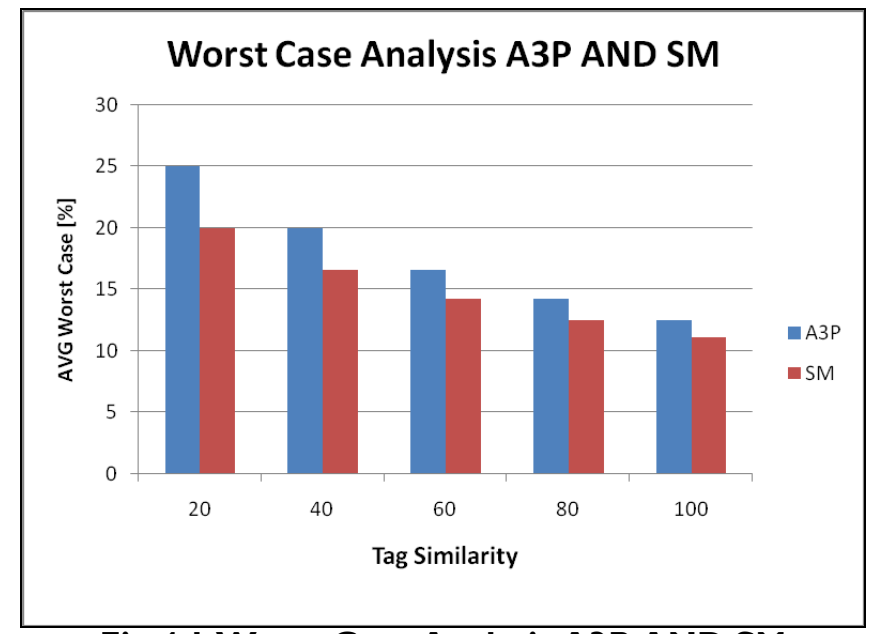

Fig 4.I Worst Case Analysis A3P AND SM

\section{User Wise Processed Result}

Table 4.2 and Fig 4.2 is describing the experimental result for user wise process result in proposed system. The table contains user id, share id count, comment count details, thread count details, and replies details count are shown below. Access Count $=$ (User Request $*$ No. of. User)

Table: 4.2

User Wise Processed Result

\begin{tabular}{|c|c|c|c|c|}
\hline USER ID & SHARE & COMMENT & THREAD & REPLIES \\
\hline I & I & 2 & I & 4 \\
\hline 2 & I & 2 & 3 & I \\
\hline 3 & 0 & I & I & 3 \\
\hline 4 & 2 & 3 & 2 & 4 \\
\hline 5 & 4 & 2 & 0 & 5 \\
\hline
\end{tabular}

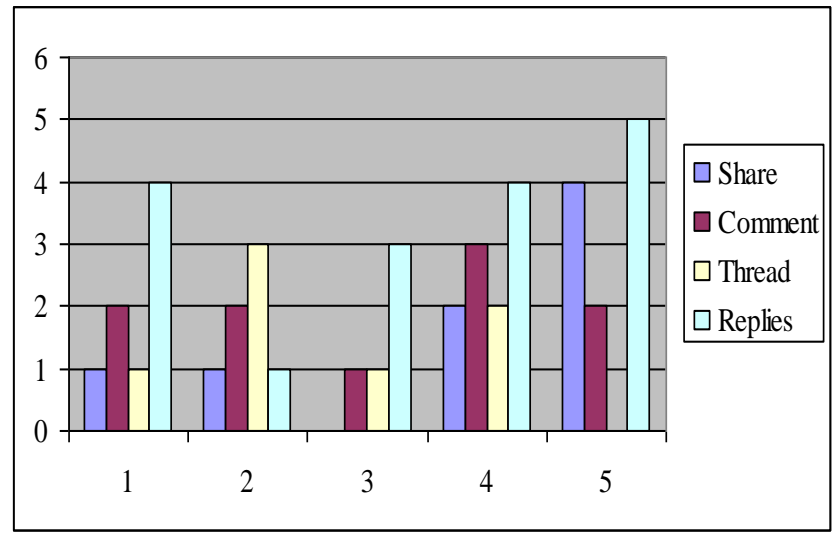

Fig 4.2 User Wise Processed Result 


\section{CONCLUSION}

In this paper assessment an Adaptive Privacy Policy Prediction (A3P) system that helps customers with motorizing the security methodology settings for their moved pictures. The A3P structure gives a careful framework to deduce security tendencies reliant on the information open for a given customer. It is moreover satisfactorily dealt with the issue of coldstart, using group environment information. The test study exhibits that the A3P is a rational instrument that offers significant redesigns over current approaches to manage insurance. It helps the customer with getting their record nuances and pictures they share. They have the decisions to set individuals who can see their information, for instance, picture and text they share. This paper consolidates the decision to set the record size limit. The customer of the record can set the report size according to their necessities. There is no convincing motivation to make the plan settings each time when the customer endorsed into the record. Modified course of action setting is included.One of these potential applications is the game plan of web access functionalities, for instance, content isolating and disclosure. For this to transform into a reality, regardless, it is imperative to expand the designing of current synergistic naming organizations to fuse a system layer that maintains the prerequisite of customer tendencies. Agreeable naming has been procuring pervasiveness, it have been become more clear the necessity for security affirmation; since marks are tricky information just as by virtue of the threat of cross alluding to.

\section{REFERENCES}

I. Abhari and M. Soraya, "Workload generation for youtube," Multimedia Tools and Applications, vol. 46, no. I, pp. 91-II8, 2017.

2. M. Barbera, J. Stefa, A. Viana, M. de Amorim, and M. Boc, "Vip delegation: Enabling vips to offload data in wireless social mobile networks," in DCOSS'II. IEEE, 2016, PP. I-8.

3. R. Gracia-Tinedo M. Conti, and A. Passarella, "Contentplace: socialaware data dissemination in opportunistic networks," in Proceedings of the IIth international symposium on Modeling, analysis and simulation of wireless and mobile systems. ACM, 2018, pp. 203-210.

4. P. Hui, P. Hui, J. Crowcroft, C. Diot, R. Gass, and J. Scott, "Impact of uman mobility on opportunistic forwarding algorithms," Mobile Computing, IEEE Transactions on, vol. 6, pp. 606-620, 2017.

5. A.J. Khan and F. Rahman, "Analyzing privacy designs of mobile social networking applications," in IEEE/IFIP EUC'08, vol. 2. IEEE, 2018, pp. 83-88.

6. A. Pietiläinen and C. Diot, "The where and when of finding new friends: Analysis of a location-based social discovery network." in ICWSM, 2013.

7. K. Thilakarathna, E. Ferrari, R. Heatherly, M. Kantarcioglu, and B.M. Thuraisingham, "Semantic Web-Based Social Network Access Control," Computers \& Security, vol. 30, pp. 108-II5, 2011.

8. V.Senthilkumar,M.Keerthivasan,R.Kishore,M.Kalathi and G,Monica "providing security against Ip crowdsourced spoofing attacks on cloud using topoguard algorithm,"South Asian Journal of Engineering and Technology, Volume 7, Issue I, PP I58 - 165, 2017.

9. Y. Cheng, J. Park, and R.S. Sandhu, "A User-to-User Relationship-Based Access Control Model for Online Social Networks," Proc. 26th Ann. IFIP WG II.3 Conf. Data and Applications Security and Privacy, 2012.

10. P. Dunphy, A.P. Heiner, and N. Asokan, "A Closer Look at Recognition-Based Graphical Passwords on Mobile Devices," Proc. Symp. Usable Privacy and Security, 2012.

II. Dwyer, S.R. Hiltz, and K. Passerini, "Trust and Privacy Concern within Social Networking Sites: A Comparison of Facebook and MySpace," Proc. Am. Conf. Information Systems (AMCIS '07), 2017.

12. L. Fang and K. LeFevre, "Privacy Wizards for Social Networking Sites," Proc. Conf. World Wide Web, 2012.

13. P.W. Fong, "Relationship-Based Access Control: Protection Model and Policy Language," Proc. Conf. Data and Application Security and Privacy, 2011.

14. S.T. Iqbal and B.P. Bailey, "Investigating the Effectiveness of Mental Workload As a Predictor of Opportune Moments for Interruption," Proc. Computer Human Interaction Extended Abstracts on Human Factors in Computing Systems (CHI '05), 2015.

15. Harrison, S. and Dourish, P. (1996) Replacing space: The roles of place and space in collaborative systems, Proceedings of CSCW 1996.

16. Schuler D., (1994) Community networks: Building a new participatory medium. CACM 37, I, 38-5 I

17. Want, R., Hopper, A., Falcao, V., Gibbons, J., (2000) The active badge location system, ACM Transactions on Information Systems, 10, I., 91-102

18. J. Bonneau and S. Preibusch, "The Privacy Jungle: On the Market for Data Protection in Social Networks," Proc. Workshop the Economics of Information Security (WEIS '09), 2010.

19. H. Krasnova, O. Gu "nther, S. Spiekermann, and K. Koroleva, "Privacy Concerns and Identity in Online Social Networks," Identity in the Information Soc., vol. 2, no. I, pp. 39-63, 2010. 
Dr. V. Senthil kumar, Mr. P. Jeevanantham, Dr. A. Viswanathan ,

Dr. Vignesh Janarthanan, Dr. M. Umamaheswari , Dr. S. Sivaprakash

20. P.A. Norberg, D.R. Horne, and D.A. Horne, "The Privacy Paradox: Personal Information Disclosure Intentions versus Behaviors," J. Consumer Affairs, vol. 4I, pp. 100-126, 201 I.

21. Dwyer, S.R. Hiltz, and K. Passerini, "Trust and Privacy Concern within Social Networking Sites: A Comparison of Facebook and MySpace," Proc. Am. Conf. Information Systems (AMCIS 'I0), 2010. 